Original paper

\title{
Evaluation of the effects of implementing a diode transmission device into the clinical workflow
}

\author{
Adam B. Paxton ", Vikren Sarkar, Jeremy N. Kunz, Martin Szegedi, Hui Zhao, Y. Jessica Huang, \\ Geoff Nelson, Prema Rassiah, Fan-Chi Frances Su, Bill J. Salter \\ University of Utah, Department of Radiation Oncology, 1950 Circle of Hope Drive, Salt Lake City, UT 84112, United States
}

\section{A R T I C L E I N F O}

\section{Keywords:}

Transmission detector

IMRT

Quality assurance

Safety

\begin{abstract}
A B S T R A C T
Purpose: This work investigated effects of implementing the Delta ${ }^{4}$ Discover diode transmission detector into the clinical workflow.

Methods: PDD and profile scans were completed with and without the Discover for a number of photon beam energies. Transmission factors were determined for all beam energies and included in Eclipse TPS to account for the attenuation of the Discover. A variety of IMRT plans were delivered to a Delta ${ }^{4}$ Phantom + with and without the Discover to evaluate the Discover's effects on IMRT QA. An imaging QA phantom was used to assess the detector's effects on MV image quality. OSLDs placed on the Phantom+ were used to determine the detector's effects on superficial dose.

Results: The largest effect on PDDs after $d_{\max }$ was $0.5 \%$. The largest change in beam profile symmetry and flatness was $0.2 \%$ and $0.1 \%$, respectively. An average difference in gamma passing rates $(2 \% / 2 \mathrm{~mm}) \mathrm{of} 0.2 \%$ was observed between plans that did not include the Discover in the measurement and calculation to plans that did include the Discover in the measurement and calculation. The Discover did not significantly change the MV image quality, and the largest observed increase in the relative superficial dose when the Discover was present was $1 \%$.

Conclusions: The effects the Discover has on the linac beam were found to be minimal. The device can be implemented into the clinic without the need to alter the TPS beam modeling, other than accounting for the device's attenuation. However, a careful workflow review to implement the Discover should be completed.
\end{abstract}

\section{Introduction}

Because of their inherent complexity, intensity-modulated radiation treatments are often validated by measurements on a phantom prior to being delivered to a patient. However, measurements of the actual delivery to the patient are uncommon. When measurements are acquired during treatment, they can be performed with point detectors, such as optically-stimulated luminescent dosimeters (OSLDs) [1], diodes [2], or similar devices $[3,4]$. However, these measurements only give information of the delivered point dose at the location of the detector, and the results are dependent on the users' placement of the detector on the patient. In addition, this location is typically superficial and is not representative of the dose to deeper targets. Electronic portal imaging devices (EPIDs) have also been used to measure exit dose during patient treatments, and have been shown to be sensitive to both machine and patient position errors [5-7]. Though, this requires the EPID to be deployed during treatment, which may not always be possible due to clearance. This would be particularly true for treatments that utilize couch rotations, such as stereotactic radiosurgery. As an alternative to measurements, machine log files can be used to compare the actual machine parameters during treatment (e.g., multileaf collimator (MLC) positions, gantry angle, etc.) to the expected parameters from the treatment plan. However, it has been observed that these log files are not always accurate $[8,9]$, and their use for validating treatment plans has been debated [10].

Transmission detectors are another measurement system that have the potential to independently verify the machine performance during a patient's treatment [11], are not dependent on proper placement by the user, and do not require the EPID to be deployed. The potential ability to be system and user independent and validate most treatment fields could be important in terms of patient safety in that unintended treatment deviations could be caught before they become detrimental to the

\footnotetext{
* Corresponding author.

E-mail address: adam.paxton@hci.utah.edu (A.B. Paxton).
} 
patient. Though, transmission detector systems are not sensitive to patient position errors, so other methods would be needed to gain this information [12]. Currently available transmission detector systems utilize either ion chambers or diodes. In general, these devices attenuate the beam in the range of approximately $1 \%$ to $8 \%$ depending on the construction of the device [13].

In a recent Point/Counterpoint article [14], concerns with implementing the use of transmission detectors were discussed and included potential collision/clearance issues, attenuation and beam modeling effects, and an increase in skin dose. Therefore, prior to introducing a transmission device into clinical use, it is important to evaluate the attenuation effects the device has on the beam and to assess how the device may alter the inherent qualities of the beam modeled in the treatment planning system (TPS) (e.g., beam energy and spectrum as indicated by the depth and cross profiles). Such changes could require a recommissioning of the beam in the TPS. Additionally, it is important to investigate the device's effects on other aspects of the clinical workflow.

The Delta ${ }^{4}$ Discover (ScandiDos AB, Uppsala, Sweden) transmission detector is a diode-based system that mounts to the head of the linear accelerator (linac) gantry. It analyzes MLC positions, gantry angle, and collimator angle and compares these to what was intended from the treatment plan. If used in combination with the Delta ${ }^{4}$ Phantom + for pre-treatment patient-specific quality assurance, it is also able to assess the dose delivered for each treatment fraction compared to the planned dose [13]. This work investigated the Discover's effects on photon beams as well as its effects on intensity-modulated radiation therapy (IMRT) quality assurance (QA), megavoltage (MV) imaging, and superficial dose.

\section{Materials and methods}

\subsection{Discover transmission detector}

The Delta ${ }^{4}$ Discover transmission diode detector consists of $4040 \mathrm{p}$ type diode detectors. The diodes have a detection area of $20 \mathrm{~cm} \times 25 \mathrm{~cm}$ projected at isocenter. The isocenter-projected spacing between the detectors is $2.5 \mathrm{~mm}$ along the direction of MLC leaf motions and $5 \mathrm{~mm}$ perpendicular to leaf motion. This covers each of the $5 \mathrm{~mm}$-wide central 40 leaf pairs of a Varian (Palo Alto, CA) Millennium 120 MLC. If a field irradiates areas outside the sensitive region of the device, these portions of the field will not be measured and evaluated. The individual diodes are disk-shaped with a $1 \mathrm{~mm}$ diameter and $0.05 \mathrm{~mm}$ thickness. According to the manufacturer's technical specifications, the diodes have a sensitivity decrease of less than $0.1 \%$ per $\mathrm{kGy}$ of delivered dose. The Discover device is wireless, communicating via Wi-Fi to the associated computer, and is powered by up to four rechargeable batteries. It attaches to the collimator of a Varian TrueBeam linac at the same attachment points as the accessory tray, as shown in Fig. 1. The device extends $2.2 \mathrm{~cm}$ from the TrueBeam collimator, and so there is a reduction in clearance. However, the device is positioned close enough to the collimator such that it does not interfere with the LaserGuard II infrared laser collision detection system of the Varian TrueBeam system, thereby maintaining the functionality of this collision detection system. The dimensions of the Discover relative to a Varian collimator are shown in Fig. 2. The Discover weighs $8.7 \mathrm{~kg}(19.1 \mathrm{lb})$ without batteries and 10.7 $\mathrm{kg}(23.5 \mathrm{lb})$ with all four batteries. All measurements with the Discover were performed on a Varian TrueBeam with Millennium 120 MLC.

\subsection{Beam scanning}

Beam scanning was performed at $100 \mathrm{~cm}$ source-to-surface distance (SSD) for the photon beam energies of 6, 6 flattening filter free (FFF), 10, $10 \mathrm{FFF}, 15$, and $18 \mathrm{MV}$. Percent depth dose (PDD) scans were taken along the central axis of the beam to $35 \mathrm{~cm}$ depth for field sizes of $(4 \times$ $4),(10 \times 10)$, and $(30 \times 30) \mathrm{cm}^{2}$ and normalized to $d_{\text {max }}$. Crossline profiles were taken for the same field sizes at $d_{\max }$ and $10 \mathrm{~cm}$ depths and normalized to the central axis of the profile. All scans were completed with and without the Discover detector in place and were performed using Sun Nuclear (Melbourne, FL) SNC125c scanning chambers and 3D Scanner water tank. The scans with and without the Discover were then compared to determine the effects the detector had on the PDDs and profiles. As shown in Fig. 2, beams that extend beyond the sensitive region (i.e., the globetop and embedded silicon) still transverse other components of the device (i.e., the carbon fiber panels, etc.), To specifically evaluate a point beyond the sensitive detection area of the Discover, points at $\pm 13 \mathrm{~cm}$ off axis were compared between $(30 \times 30)$ $\mathrm{cm}^{2}$ scans with and without the Discover.

\subsection{Transmission measurements}

To evaluate beam transmission through the Discover detector, a 0.3 $\mathrm{cm}^{3}$ Semiflex ionization chamber (PTW-Freiburg) was positioned in a Solid Water (Gammex, Middleton, WI) block phantom at $10 \mathrm{~cm}$ depth. The phantom was set up at a $90 \mathrm{~cm}$ SSD. Charge readings from the ion chamber were collected with and without the Discover detector in place for all photon beam energies. The transmission factor for each beam energy was taken as the ratio of the average charge measurement with the Discover in place to the average charge measurement without the Discover. This was completed for field sizes of $(4 \times 4),(10 \times 10)$, and $(30 \times 30) \mathrm{cm}^{2}$. The average percent attenuation was calculated as $[100 \% \times(1-$ average transmission $)]$ for each beam energy.

In order to account for the attenuation of the Discover in treatment plans, the transmission factors from the $(10 \times 10) \mathrm{cm}^{2}$ field size for each beam energy were entered into Eclipse version 15.5 (Varian) TPS as a tray factor.

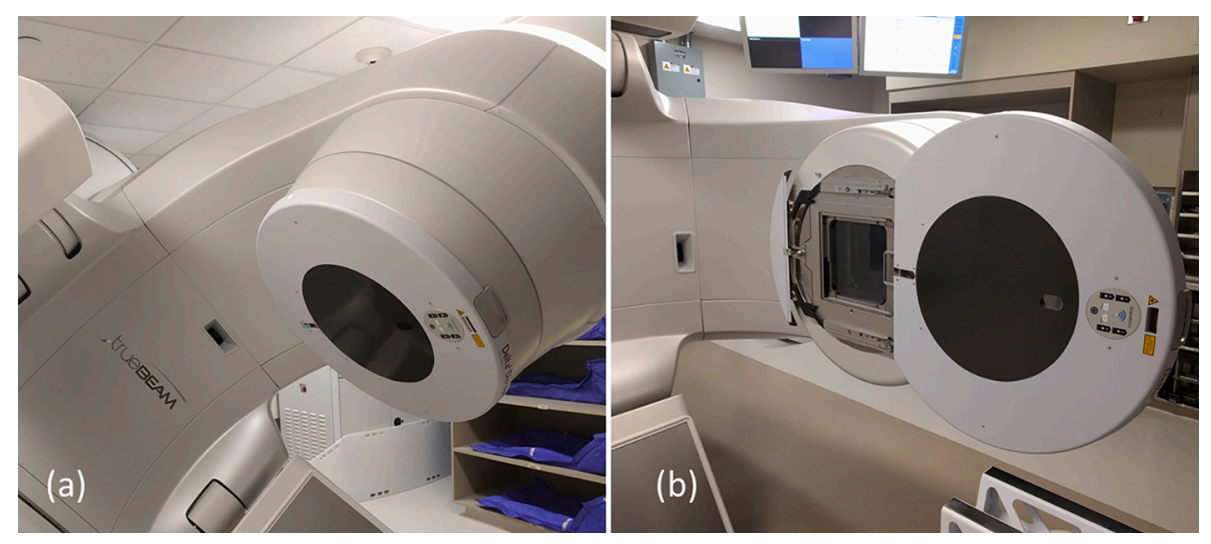

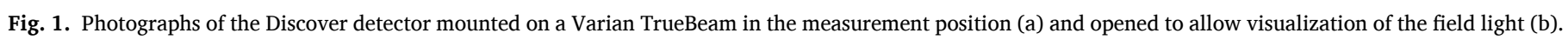




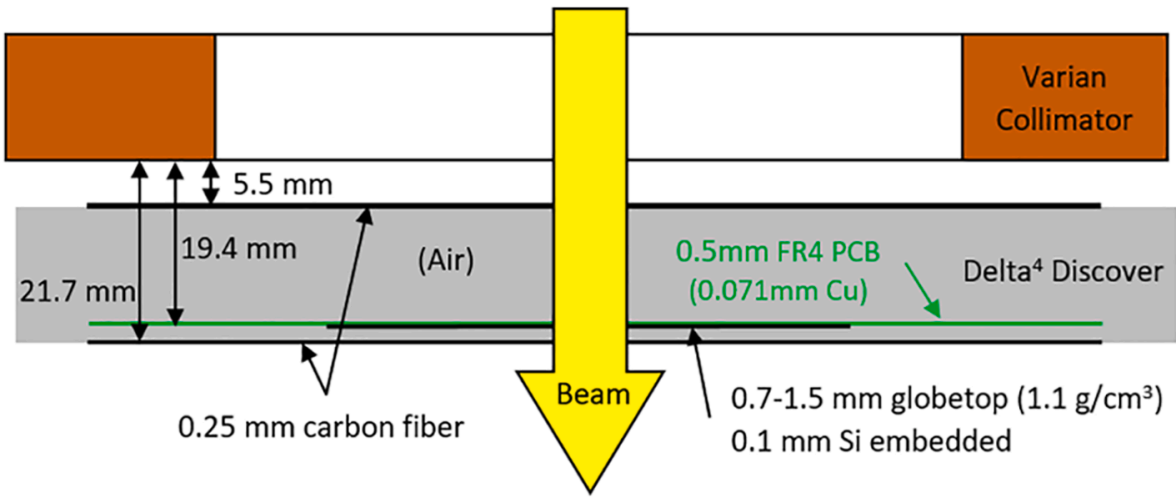

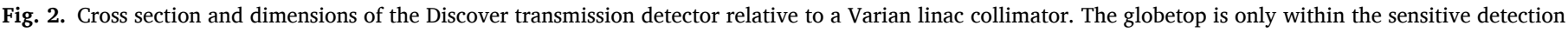
area of the Discover. (Dimensions provided by ScandiDos. Drawing is not to scale.)

\subsection{Effects of the detector on $M V$ imaging}

The Discover device is ideally intended to remain in place for all patient treatments on a given linac, except those that would not support it being in place (e.g., an electron treatment that requires an electron cone or a cone-based radiosurgery treatment). This means the Discover would be in place during MV imaging. To assess the Discover's effects on MV image quality, six $6 \mathrm{MV}$ images with and without the Discover were acquired with a Standard Imaging (Middleton, WI) QC-3 imaging phantom on the image panel. The imager height was set to $150 \mathrm{~cm}$ source-to-imager distance (SID). This SID is the default position used clinically. The images were analyzed using TotalQA (Image Owl, Greenwich, NY) and the metrics of spatial resolution and contrast-tonoise ratio (CNR) were compared between images acquired with the Discover to images acquired without the Discover. Spatial resolution is reported as the spatial frequency $(\mathrm{lp} / \mathrm{mm}$ ) at $50 \%$ (f50) of the relative modulation transfer function.

\subsection{Effects of the detector on IMRT delivery validation}

To assess the effect of the Discover on IMRT delivery validation, a variety of IMRT plans were delivered to a Delta ${ }^{4}$ Phantom $+[15,16]$ with and without the Discover mounted to the head of the gantry. The plans were selected to represent the variety of plan types delivered in a radiation oncology clinic. The details of the evaluated plans are given in Table 1. Calculations from Eclipse with and without the attenuation of the Discover accounted for were compared to their respective measurement. Gamma analyses using $2 \%$ dose deviation and $2 \mathrm{~mm}$ distance-

Table 1

Treatment plan details of the VMAT/IMRT plans that were evaluated.

\begin{tabular}{|c|c|c|c|c|}
\hline Plan Site & $\begin{array}{l}\text { Beam } \\
\text { Energy } \\
\text { (MV) }\end{array}$ & $\begin{array}{l}\text { Delivery } \\
\text { Technique }\end{array}$ & $\begin{array}{l}\text { Dose }(\mathrm{cGy}) \\
\times \text { Fractions }\end{array}$ & $\begin{array}{l}\text { Superficial } \\
\text { Dose } \\
\text { Evaluated }\end{array}$ \\
\hline Pelvis (Male) & 10 & 3 arc VMAT & $180 \times 28$ & $\mathrm{X}$ \\
\hline $\begin{array}{l}\text { Head and Neck } \\
\text { (SIB) }\end{array}$ & 6 & 3 arc VMAT & $\begin{array}{l}200,180 \times \\
30\end{array}$ & $\mathrm{X}$ \\
\hline Prostate SBRT & $10 \mathrm{FFF}$ & 2 arc VMAT & $725 \times 5$ & $\mathrm{X}$ \\
\hline Spine SBRT & $6 \mathrm{FFF}$ & 2 arc VMAT & $800 \times 3$ & $\mathrm{X}$ \\
\hline $\begin{array}{l}\text { Brainstem } \\
\text { (Ependymoma) }\end{array}$ & 6 & 9 field IMRT & $180 \times 30$ & \\
\hline Brain & 10 & 4 arc VMAT & $180 \times 30$ & \\
\hline Pelvis (Female) & 10 & 9 field IMRT & $180 \times 25$ & \\
\hline $\begin{array}{l}\text { Pelvis (Male) } \\
\quad \text { (SIB) }\end{array}$ & 10 & 2 arc VMAT & $\begin{array}{l}240,180 \times \\
28\end{array}$ & $\mathrm{X}$ \\
\hline Pituitary Fossa & 6 & 4 arc VMAT & $180 \times 24$ & $\mathrm{X}$ \\
\hline Scalp & 6 & 9 field IMRT & $250 \times 20$ & $\mathrm{X}$ \\
\hline
\end{tabular}

FFF - flattening filter free; SIB - simultaneous integrated boost; SBRT - stereotactic body radiation therapy; IMRT - intensity modulated radiation therapy; VMAT - volumetric modulated arc therapy to-agreement criteria were used to compare the measured and calculated dose distributions. The $2 \% / 2 \mathrm{~mm}$ criteria was selected for the gamma analyses because it was the standard criteria of the investigating institution and because it would be more sensitive to differences than less stringent criteria (e.g., $3 \% / 2 \mathrm{~mm}$ or $3 \% / 3 \mathrm{~mm}$ ). Global normalization and a $10 \%$ dose threshold were used for all analyses. The gamma analyses for the two scenarios (calculation and measurement include the Discover vs. calculation and measurement do not include the Discover) were compared for each treatment plan.

\subsection{Superficial dose measurements}

Of the plans that were used to evaluate the Discover's effect on IMRT delivery validation, a subset was used to evaluate the detector's effect on superficial dose (see Table 1). Changes in superficial dose were determined by placing nanoDot OSLDs [17] (Landauer, Glenwood, IL) on the anterior surface of the Delta ${ }^{4}$ Phantom + and delivering plans with and without the Discover in place. One OSLD measurement was completed for each scenario. The location of the OSLD on the Delta ${ }^{4}$ Phantom + was outlined to ensure reproducible placement and orientation of the dosimeters for all measurements. These measurements were completed prior to the attenuation of the Discover being accounted for in the TPS as a tray factor. Since the same plan delivery was used for measurements with and without the Discover (i.e., both plan deliveries had the same number of monitor units), the OSLD measurement results with the Discover in place were scaled up by the beam-energy-specific transmission factors determined in this study (as described in Section II C). The OSLD measurement results with and without the Discover were compared for each plan to determine the effects on superficial dose.

\section{Results}

\subsection{Beam scanning}

There was good agreement between the PDDs with and without the Discover for all energies, with the largest difference for any beam, any field size, at any depth point on the curve being $0.5 \%$ after $\mathrm{d}_{\max }$. The PDD values with and without the Discover at $10 \mathrm{~cm}$ and $20 \mathrm{~cm}$ depths were within $0.4 \%$ across all beam energies. The depth of $d_{\max }$ was changed by a maximum of $0.1 \mathrm{~cm}$ for all energies. Likewise, the beam profiles with and without the Discover agreed well, with the largest differences in flatness of $0.1 \%$ and symmetry of $0.2 \%$. The maximum detected field size change was $0.1 \mathrm{~mm}$ and the maximum observed change in penumbra $(80 \%$ to $20 \%)$ was $0.2 \mathrm{~mm}$. For the $(30 \times 30) \mathrm{cm}^{2}$ scans, the average difference between points $\pm 13 \mathrm{~cm}$ off axis with and without the Discover was $0.1 \%$. The maximum difference was $0.3 \%$. 


\subsection{Transmission measurements}

The results of the transmission measurements are given in Table 2 . The average attenuation of the Discover device ranged from $0.8 \%$ to $1.6 \%$ for 18 and 6 FFF beams, respectively. As seen in Table 2, the transmission factor differences between the field sizes are minimal. For all beam energies, the largest difference in transmission factor relative to the $(10 \times 10) \mathrm{cm}^{2}$ field size was $0.2 \%$. As described previously, the transmission factors for the $(10 \times 10) \mathrm{cm}^{2}$ field size were included in Eclipse TPS as tray factors for each beam energy to account for the attenuation of the device in plan calculations.

\subsection{Effects of the detector on MV imaging}

The average (standard deviation) of f50 with and without the Discover in place was $0.441(0.003) \mathrm{lp} / \mathrm{mm}$ and $0.439(0.004) \mathrm{lp} / \mathrm{mm}$, respectively. The average (standard deviation) of the CNR with and without the Discover was 187.4 (6.3) and 191.6 (4.3), respectively. The spatial resolution and CNR results acquired with the Discover in place were within one standard deviation of the results without the Discover. Based on these results, the presence of the Discover device during MV imaging was found to have no significant impact on the quality of MV images. Qualitatively, the subcomponents of the Discover device were not visible in the images acquired with the Discover mounted on the linac.

\subsection{Effects of the detector on IMRT delivery validation}

The gamma pass rates for the two scenarios (measurement and calculation with the Discover or measurement and calculation without the Discover) are given in Fig. 3 for all of the evaluated treatment plans. There is very good agreement between the gamma pass rates for these two scenarios, with the average (standard deviation) difference in pass rate from the current to the new process being $0.2 \%(1.1 \%)$ and the maximum difference being $-2.1 \%$ (for the prostate SBRT plan). These results suggest changing the clinical process from not using the Discover to including the Discover should minimally affect IMRT delivery validation results.

\subsection{Superficial dose measurements}

Fig. 4 shows the relative superficial dose with and without the Discover for a variety of treatment plans. For all plans, the superficial dose measured with the Discover in place was higher than without the Discover in place. The largest increase in relative superficial dose was $1 \%$ for the scalp plan, and the average increase was $0.4 \%$. Therefore, the increase in superficial doses were minimal when the Discover was in place during treatment delivery for the measured IMRT plans.

\section{Discussion}

Li et al. [13] previously investigated a prototype of the clinically-

\section{Table 2}

Transmission factors and attenuation of the Delta ${ }^{4}$ Discover detector for various photon energies and field sizes.

\begin{tabular}{llllll}
\hline & \multicolumn{4}{c}{ Transmission Factor } & \\
\cline { 2 - 5 } $\begin{array}{l}\text { Energy } \\
\text { (MV) }\end{array}$ & $\begin{array}{l}(4 \times 4) \\
\mathrm{cm}^{2}\end{array}$ & $\begin{array}{l}(10 \times 10) \\
\mathrm{cm}^{2}\end{array}$ & $\begin{array}{l}(30 \times 30) \\
\mathrm{cm}^{2}\end{array}$ & $\begin{array}{l}\text { Average } \\
\text { Average } \\
\text { Attenuation (\%) }\end{array}$ \\
\hline 6 & 0.987 & 0.987 & 0.988 & 0.987 & 1.3 \\
6FFF & 0.982 & 0.984 & 0.985 & 0.984 & 1.6 \\
10 & 0.989 & 0.989 & 0.991 & 0.990 & 1.0 \\
$10 \mathrm{FFF}$ & 0.988 & 0.988 & 0.989 & 0.988 & 1.2 \\
15 & 0.990 & 0.990 & 0.992 & 0.991 & 0.9 \\
18 & 0.991 & 0.991 & 0.993 & 0.992 & 0.8 \\
\hline
\end{tabular}

released Discover detector. In short, the device they investigated was positioned farther from the linac head (i.e., closer to the patient) and the dimensions of some components were different. One benefit of the clinically-released version being closer to the linac (extending $2.2 \mathrm{~cm}$ vs. $5.0 \mathrm{~cm}$ from the collimator) is that the chances of collisions are reduced. All of the specific dimensional differences can be seen by comparing Fig. 2 in this work with Fig. 1 from Li et al. The average transmission factors that Li et al. determined for 6 and $15 \mathrm{MV}$ were 0.989 and 0.993 , respectively. These are very similar to this work's determined values of 0.987 and 0.991 , respectively, demonstrating that the redesign of the clinically-released Discover had minimal effects on the transmission of the device. Additionally, this work determined transmission factors for the other photon energies listed in Table 2, which include all of the commonly used photon energies on the TrueBeam platform. These results can serve as a reference for future users of the Discover device using the Varian TrueBeam platform.

Based on the good agreement between the PDD and cross profile scans with and without the Discover, the beam energy is minimally affected by the device and the device uniformly attenuates the beam across the field. Therefore, no adjustments to the beam models should be needed and only accounting for the central axis attenuation of the device in the TPS calculation should be sufficient. As shown in Fig. 3, good agreement was found in the gamma passing rate between scenarios where both calculation and measurement did not include the Discover (representative of current clinical workflow) to scenarios where calculations and measurements both included the Discover (representative of a new workflow in which the Discover is in place during treatments). The agreement between these results further validates the transmission factors applied in the TPS calculations alone appropriately account for the Discover device.

This work found a minimal increase in superficial dose when the Discover device was used (Fig. 4). This study investigated a variety of IMRT treatment plans that included a range of field sizes (large - such as pelvis and head and neck treatments, to small - such as partial brain and prostate SBRT), but all superficial dose measurements were completed with the Delta ${ }^{4}$ Phantom + centered at isocenter. This results in an SSD of $89 \mathrm{~cm}$. For larger open field sizes and smaller SSDs, the relative increase in superficial dose with the Discover in place would likely go up. Cheung et al. [18] reported the effects of superficial dose going up with increasing field sizes and decreasing SSDs with the Dolphin transmission device for open (non-IMRT) fields. Likewise, Li et al. [13] determined the Discover prototype caused an increase in the surface dose relative to $\mathrm{d}_{\max }$ of $8 \%$ and $9 \%$ for $6 \mathrm{MV}$ and $15 \mathrm{MV}$, respectively, with an open field size of $(40 \times 40) \mathrm{cm}^{2}$. Due to the dynamic delivery nature of IMRT, these treatments would be those that would benefit most from the independent measurement provided by the Discover, so those were the treatments that were evaluated in this work. Even conformal (non-IMRT) treatments rarely utilize field sizes that approach $(40 \times 40) \mathrm{cm}^{2}$. However, if from a practicality or safety standpoint, the Discover is going to be used for all treatments, then a case-by-case evaluation may be needed for large field conformal treatments with smaller SSDs. These evaluations can help physicians determine if the potential benefits of the Discover are outweighed by the increase in superficial dose.

From the perspective of the American Association of Physicists in Medicine (AAPM) Task Group 100 report (TG-100) [19], an analysis of a workflow should try to identify the highest risk failure modes and alter the workflow to help mitigate the risk. TG-100 also discusses that each clinic is expected to have unique processes due to a number of common variations (e.g., oncology information system, linac vendor, TPS, etc.). However, with regard to transmission detectors, an obvious failure mode would be not having the detector in place during treatment when it was planned to be in place (or vice versa), and it is worth discussing some points-to-consider related to this failure mode. Some of the following considerations are general to all transmission detectors, while others are specific to our institution's environment.

One potential idealized solution would be to use a transmission 


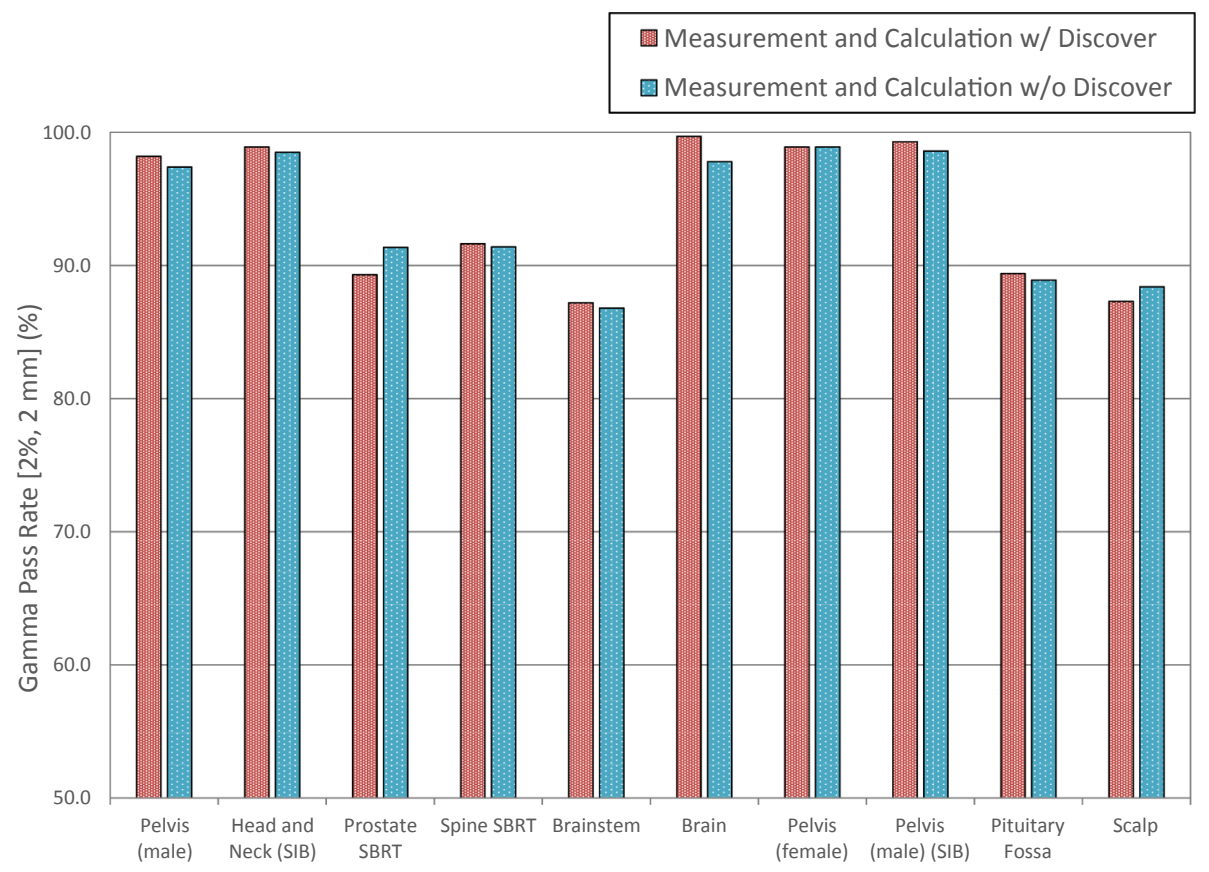

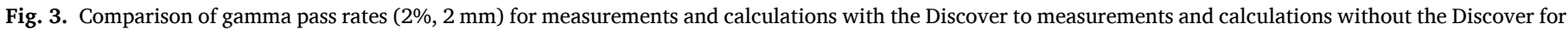
a variety of treatment plan types.

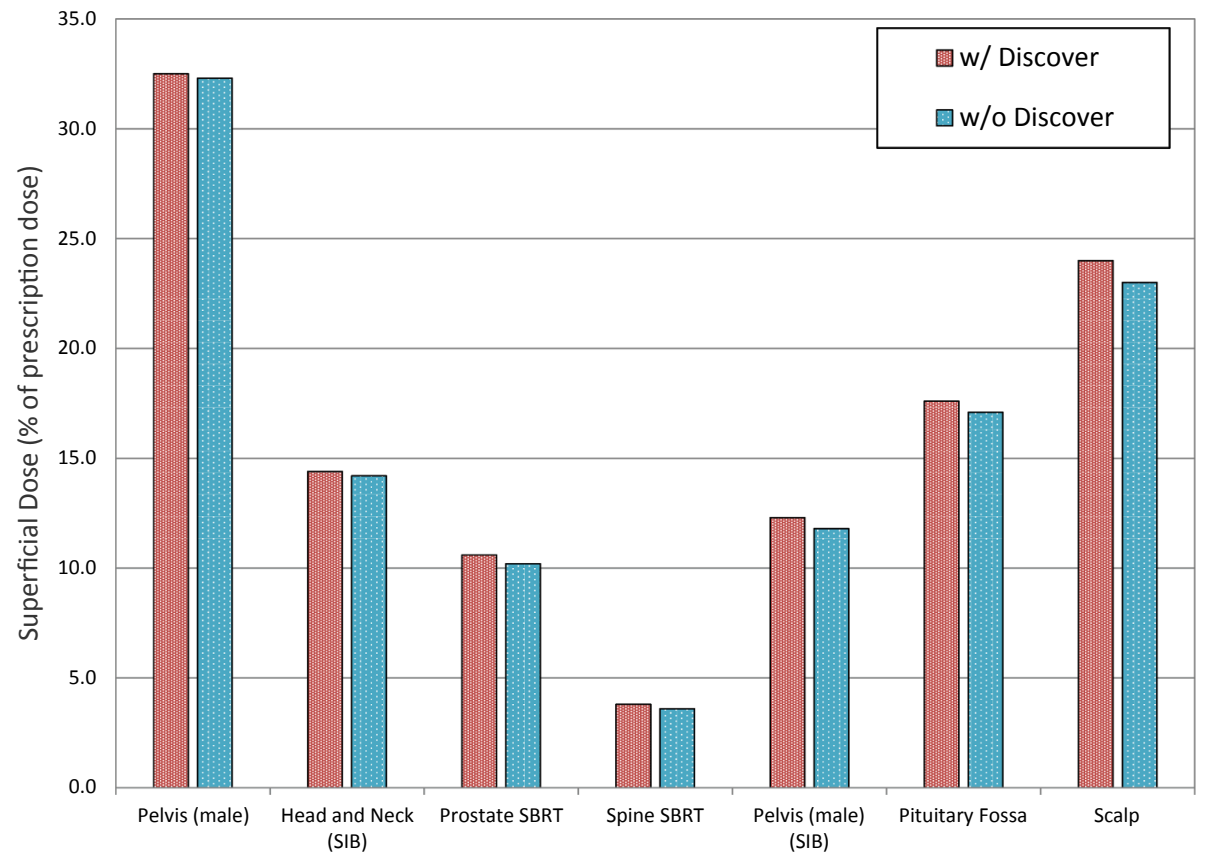

Fig. 4. Comparison of the relative OSLD-measured superficial dose with and without the Discover detector in place for a variety of treatment plan types.

detector for all patients. For this scenario, the only time the Discover would need to be dismounted would be for treatments that prohibit its use, such as electron or cone-based radiosurgery treatments. These treatments could potentially be grouped at the beginning or end of the day for efficiency sake. However, during the time that the device is being phased into the clinical workflow, this scenario may not be practical. It is necessary to design clinical workflows that allow the radiation oncology staff know if the device should be present or not.

Eclipse TPS (version 15.5) allows for a unique accessory code for the Discover device to indicate it should be present during treatment. However, the authors' institution uses Mosaiq (version 2.64) (Elekta,
Stockholm, Sweden) as their oncology information system. For this configuration, the unique code does not transfer with the plan import from Eclipse to Mosaiq, and must be entered manually. In this example, there is a higher potential for this unintended situation to occur. Each clinic will need to evaluate their specific processes to identify any potential situations that could increase the likelihood of the failure mode.

$\mathrm{Li}$ et al. [13] also presented a comparison of the characteristics of various transmission detectors reported in the literature (their Table 1). Attenuation of $6 \mathrm{MV}$ beams along the central axis for the various detectors they compared ranged from $1.0 \%$ to $7.2 \%$, with ion-chamberbased devices typically having higher attenuation (ranging from 3.3\% 
to $7.2 \%$ ) and diode transmission devices typically having lower attenuation $(\sim 1 \%)$. Another available transmission detector not included in the comparison by Li et al. [13] is the Dolphin detector (IBA Dosimetry, Schwarzenbuck, Germany) $[18,20,21]$. The Dolphin is a 2D array of 1513 plane-parallel ion chambers. For the sake of reference, Cheung et al. [18] determined the attenuation of the Dolphin to be $7.8 \%$ for a 6 MV beam with a $(10 \times 10) \mathrm{cm}^{2}$ field size. Using the terminology from TG-100 [19], the severity of treating without the Discover when it was intended would be low since the attenuation is $\sim 1 \%$. In contrast, the severity of failing to include an ion-chamber-based transmission detector would be higher due to the greater attenuation. Regardless of severity, it is ideal to have processes in place that prevent the unintended setup.

IMRT QA has the potential to detect differences between the delivered and intended doses, such as those that may occur if in-beam devices, such as transmission detectors, are not present when intended. However, given that the attenuation of the Discover is $\sim 1 \%$, pretreatment IMRT QA with a commonly used criteria of 3\% / $2 \mathrm{~mm}$ [22] may not give the radiation oncology staff any indication (i.e., a failing result) that there is an unintended situation. Conversely, other transmission devices that utilize ion-chamber arrays, such as the Dolphin, have higher attenuation [18]. Therefore, not including the device during IMRT QA when it was intended would likely cause a noticeable drop gamma pass rate, and IMRT QA may be a potential method to increase the detectability of the unintended scenarios for these devices.

Simple methods such as setup notes can help communicate the intent to include a transmission detector during treatment. Another method that was introduced at the authors' institution was to add the Discover device to the list of treatment accessories within the Varian Identify system (version 2.1). Identify uses radiofrequency identification (RFID) tags on treatment accessories (such as immobilization devices, bolus, etc.) to detect their presence at the time of treatment. When a device is detected during treatment, a green checkmark appears next to the accessory. Otherwise the device is shown as missing and is indicated by a question mark. This detection functionality of Identify was extended to the Discover by placing a RFID on the device and adding it to the list of treatment accessories when it was intended to be used for treatment. This provides therapists with an indication that the Discover should be present for a given treatment, and is an example of leveraging other technology available in the clinic to help mitigate a potential failure mode.

This work sought to answer the question of how including the Discover in the beam during treatment would affect the clinical workflow and ultimately the patient dose. Overall, the effects were determined to be minimal. After an institution validates a Discover transmission detector, much of the work will be in determining the proper clinic-specific workflows to ensure patient safety. This work did not attempt to investigate the ability of the Discover device to detect errors, but this was evaluated in another publication from our group [23].

\section{Conclusions}

The effects of including the Discover transmission detector during treatment were determined to be minimal. Beam scanning results suggested that only accounting for the attenuation of the device should be sufficient. Good agreement between the IMRT QA results for the scenario where the device is not included in the measurement or the calculation (representing the workflow without the Discover) to scenario where the device is included in the measurement and the calculation (representing the workflow with the Discover) validate the transmission factors included in the TPS. However, as discussed, there are potential processes that could result in unintentionally not including the Discover during treatment. Therefore, careful attention should be paid to developing new processes that are specific to the clinic's environment when implementing the use of the Discover to ensure these unintended situations do not occur.

\section{Acknowledgements}

The authors thank Mikael Palm from ScandiDos for many helpful discussions regarding the Discover device.

\section{References}

[1] Riegel AC, Chen Y, Kapur A, Apicello L, Kuruvilla A, Rea AJ, et al. In vivo dosimetry with optically stimulated luminescent dosimeters for conformal and intensity-modulated radiation therapy: a 2-year multicenter cohort study. Pract Radiat Oncol 2017;7:e135-44. https://doi.org/10.1016/j.prro.2016.09.001.

[2] Kadesjö N, Nyholm T, Olofsson J. A practical approach to diode based in vivo dosimetry for intensity modulated radiotherapy. Radiother Oncol 2011;98:378-81. https://doi.org/10.1016/j.radonc.2010.12.018.

[3] Archambault L, Briere TM, Pönisch F, Beaulieu L, Kuban DA, Lee A, et al. Toward a real-time in vivo dosimetry system using plastic scintillation detectors. Int $\mathrm{J}$ Radiat Oncol Biol Phys 2010;78:280-7. https://doi.org/10.1016/j.ijrobp.2009.11.025.

[4] Venkataraman S, Malkoske K, Jensen M, Nakonechny K, Asuni G, Mccurdy B. The influence of a novel transmission detector on $6 \mathrm{MV}$ X-ray beam characteristics. Phys Med Biol 2009;36:2603. https://doi.org/10.1118/1.3181834.

[5] Zhuang AH, Olch AJ. Sensitivity study of an automated system for daily patient QA using EPID exit dose images. J Appl Clin Med Phys 2018;19:114-24. https://doi. org $/ 10.1002 / \mathrm{acm} 2.12303$

[6] Ricketts K, Navarro C, Lane K, Moran M, Blowfield C, Kaur U, et al. Implementation and evaluation of a transit dosimetry system for treatment verification. Phys Medica 2016;32:671-80. https://doi.org/10.1016/j.ejmp.2016.04.010.

[7] Mijnheer B, Jomehzadeh A, González P, Olaciregui-Ruiz I, Rozendaal R, Shokrani P, et al. Error detection during VMAT delivery using EPID-based 3D transit dosimetry. Phys Medica 2018;54:137-45. https://doi.org/10.1016/j. ejmp. 2018.10.005.

[8] Agnew A, Agnew CE, Grattan MWD, Hounsell AR, McGarry CK. Monitoring daily MLC positional errors using trajectory log files and EPID measurements for IMRT and VMAT deliveries. Phys Med Biol 2014:59. https://doi.org/10.1088/00319155/59/9/N49.

[9] Neal B, Ahmed M, Kathuria K, Watkins T, Wijesooriya K, Siebers J. A clinically observed discrepancy between image-based and log-based MLC positions. Med Phys 2016;43:2933-5. https://doi.org/10.1118/1.4949002.

[10] Childress NL, Chen Q, Rong Y. Parallel / Opposed: IMRT QA using treatment log files is superior to conventional measurement-based method. J Appl Clin Med Phys 2015;16:4-7. https://doi.org/10.1120/jacmp.v16i1.5385.

[11] Thoelking J, Fleckenstein J, Sekar Y, Boggula R, Lohr F, Wenz F, et al. Patientspecific online dose verification based on transmission detector measurements. Radiother Oncol 2016;119:351-6. https://doi.org/10.1016/j.radonc.2016.04.003.

[12] Esposito M, Villaggi E, Bresciani S, Cilla S, Falco MD, Garibaldi C, et al. Estimating dose delivery accuracy in stereotactic body radiation therapy: A review of in-vivo measurement methods. Radiother Oncol 2020;149:158-67. https://doi.org/ 10.1016/j.radonc.2020.05.014.

[13] Li T, Wu QJ, Matzen T, Yin FF, O'Daniel JC. Diode-based transmission detector for IMRT delivery monitoring: a validation study. J Appl Clin Med Phys 2016;17: 235-44. https://doi.org/10.1120/jacmp.v17i5.6204.

[14] Rangaraj D, Yaddanapudi S, Cai J. Transmission detectors are safe and the future for patient-specific QA in radiation therapy. Med Phys 2019;46:1-4. https://doi. org/10.1002/mp.13201.

[15] Sadagopan R, Bencomo JA, Martin RL, Nilsson G, Matzen T, Balter PA. Characterization and clinical evaluation of a novel IMRT quality assurance system. J Appl Clin Med Phys 2009;10:104-19. https://doi.org/10.1120/jacmp. v10i2.2928.

[16] Bedford JL, Lee YK, Wai P, South CP, Warrington AP. Evaluation of the Delta4 phantom for IMRT and VMAT verification. Phys Med Biol 2009:54. https://doi. org/10.1088/0031-9155/54/9/N04.

[17] Kry SF, Alvarez P, Cygler JE, DeWerd LA, Howell RM, Meeks S, et al. AAPM TG 191: clinical use of luminescent dosimeters: TLDs and OSLDs. Med Phys 2020;47: e19-51. https://doi.org/10.1002/mp.13839.

[18] Cheung JP, Perez-Andujar A, Morin O. Characterization of the effect of a new commercial transmission detector on radiation therapy beams. Pract Radiat Oncol 2017;7:e559-67. https://doi.org/10.1016/j.prro.2017.04.001.

[19] Huq MS, Fraass BA, Dunscombe PB, Gibbons JP, Ibbott GS, Mundt AJ, et al. The report of Task Group 100 of the AAPM: application of risk analysis methods to radiation therapy quality management. Med Phys 2016;43:4209. https://doi.org/ 10.1118/1.4947547.

[20] Giglioli FR, Gallio E, Franco P, Badellino S, Ricardi U, Fiandra C. Clinical evaluation of a transmission detector system and comparison with a homogeneous 3D phantom dosimeter. Phys Medica 2019;58:159-64. https://doi.org/10.1016/j. ejmp.2019.01.016. 
[21] Park SY, Park JM, Kim J, Lee S, Choi CH. Validation of new transmission detector transmission factors for online dosimetry: an experimental study. Radiat Oncol 2018;13:1-9. https://doi.org/10.1186/s13014-018-1106-y.

[22] Moyed M, Arthur O, Dimitris M, Jean M, Todd P, Andrea M, et al. Tolerance limits and methodologies for IMRT measurement-based verification QA:
Recommendations of AAPM Task Group No. 218. Med Phys 2018;45. https://doi. org $/ 10.1002 / \mathrm{mp} .12810$

[23] Sarkar V, Paxton A, Kunz J, Szegedi M, Nelson G, Rassiah-Szegedi P, et al. A systematic evaluation of the error detection abilities of a new diode transmission detector. J Appl Clin Med Phys 2019. https://doi.org/10.1002/acm2.12691. 